\title{
Creating Color Image Features Using Local Contrast Method
}

\author{
Ayman Al-Rawashdeh, Ziad Al-Qadi \\ Al-Balqa Applied University/Faculty of Engineering Technology, Department of Computor Engineering, Amman, Jordan
}

\begin{tabular}{l} 
Article Info \\
\hline Article history: \\
Received May 04, 2018 \\
Revised Aug 12, 2018 \\
Accepted Aug 21, 2018 \\
\hline Keywords: \\
Artificial Neural Network \\
Feature extraction \\
Image generation \\
Image processing \\
Image quality \\
\hline
\end{tabular}

\begin{abstract}
Digital color images are now one of the most popular data types used in the digital processing environment. Color image recognition plays an important role in many vital applications, which makes the enhancement of image recognition or retrieval system an important issue. Using color image pixels to recognize or retrieve the image, but the issue of the huge color image size that requires accordingly more time and memory space to perform color image recognition and/or retrieval. In the current study, image local contrast was used to create local contrast victor, which was then used as a key to recognize or retrieve the image. The proposed local contrast method was properly implemented and tested. The obtained results proved its efficiency as compared with other methods.
\end{abstract}

Copyright $\odot 2018$ Institute of Advanced Engineering and Science. All rights reserved.

\section{Corresponding Author:}

Ayman Al-Rawashdeh,

Al-Balqa Applied University/Faculty of Engineering Technology,

Department of Electrical Engineering, Amman, Jordan

Email: dr.ayman.rawashdeh@bau.edu.jo

\section{INTRODUCTION}

\subsection{Color Image Features}

Color image features play an important role in image manipulation, as they can be used as a finger print for fast image retrieval or image recognition [1], [2]. The process of image retrieval depends on the features that can be automatically extracted from the images themselves [3]. Feature extraction process generates features to be used in the selection and classification tasks for image retrieval process. It transforms rich content of images into various content features. This process involves the selection of features that assist in discrimination of an image. Feature selection reduces the number of features provided to the classification task. Feature extraction is considered to be most critical task among other image processing tasks. This is because the selection of the particular features for discrimination directly influences the efficacy of the image recognition and identification. Feature extraction process ends with a set of features, commonly referred to as the feature vector, which constitutes the unique representation of the image [3]. The feature victor is usually small in size as compared to the original image size, which means that training time of artificial neural network (ANN) will be reduced when image features is used to identify the image [4-7]. The ANN image identification is illustrated in Figure 1.

Reducing the data needed to identify the image leads to the optimal ANN construction [7]. The main requirements needed for an optimal ANN construction includes:

- Minimum memory space to store ANN.

- Minimum ANN input data set size.

- Minimum ANN architecture.

- $100 \%$ recognition or identifying ratio.

- Minimum ANN training time.

- Minimum image retrieving time. 
Colors image are usually represented by a histogram, which is a three columns victor [1], [2]. The histogram can be used as a signature to identify color image. Each histogram victor is a features array that is very small in size comparing with color image size $\left(256^{*} 3\right)$. Figure 2 shows a colored image with its corresponding histogram victor.
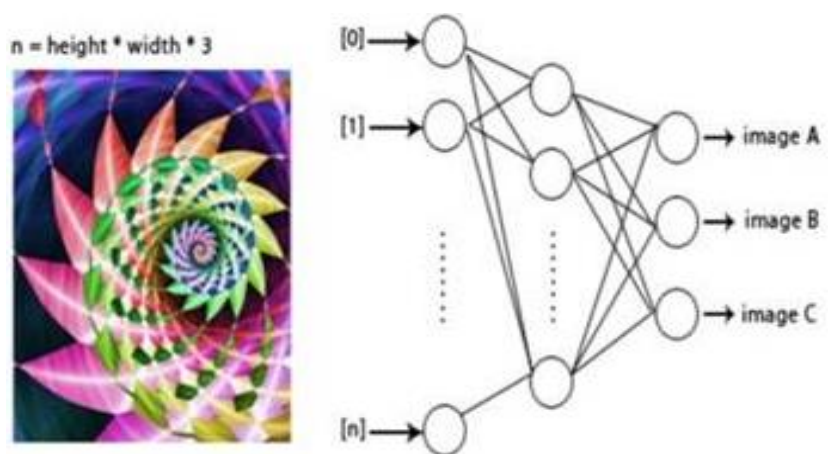

Figure 1. The ANN to identify the image pixel by pixel
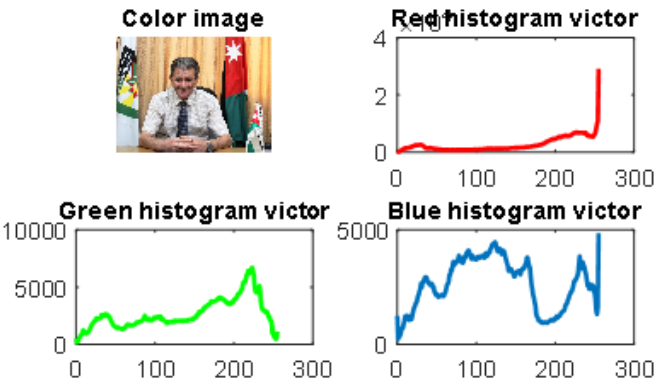

Figure 2. Histogram victors for color image

Digital color image is a 3D matrix as shown in Figure 3. Each column represents a color channel, for instance, the first column represents the red color, and the second represents the green color, while the third one represents the blue color [8-11].

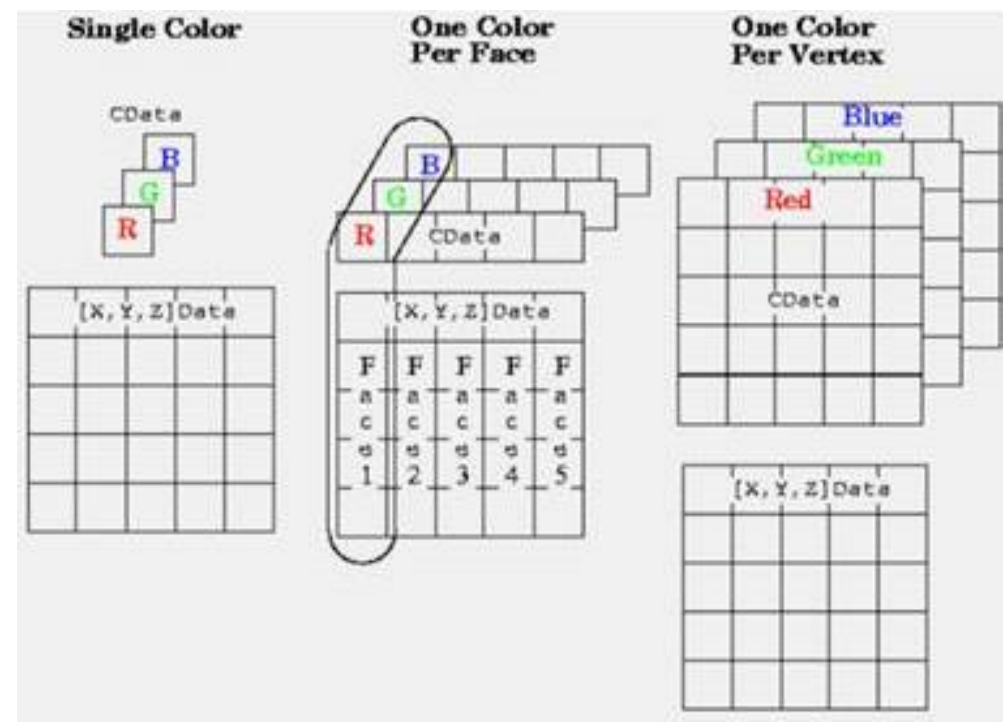

Figure 3. 3D color matrix

Each color component can be processed by a 2D matrix as shown in Figure 4 [12], [13]. Color images usually have big sizes and the identification of image pixel by pixel needs big efforts and might be considered time consuming process. This suggests the need for a more efficient method to identify color image depending on the extraction of features with a small size [8-10]. 


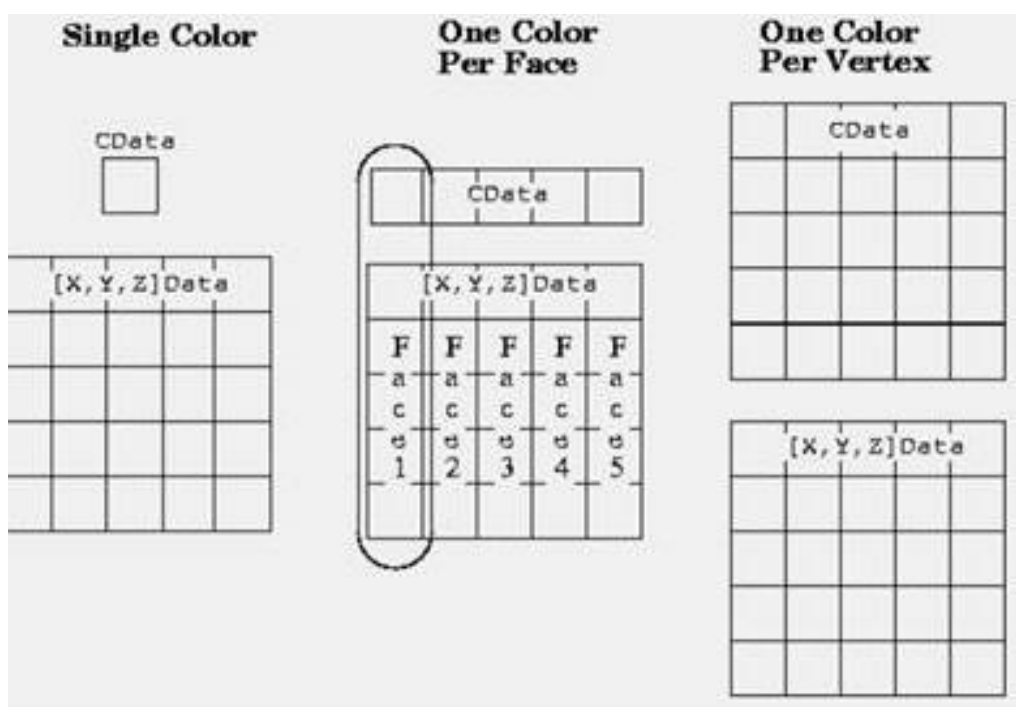

Figure 4. 2D matrix for each color

Color image can be represented by color histograms. Again, each histogram is a one column array that contains 256 elements. The histogram can be used as image identifier, and accordingly, image identification and retrieval might be enhanced leading to a quick system response time [14], [15] as could be deduced from Figure 5.
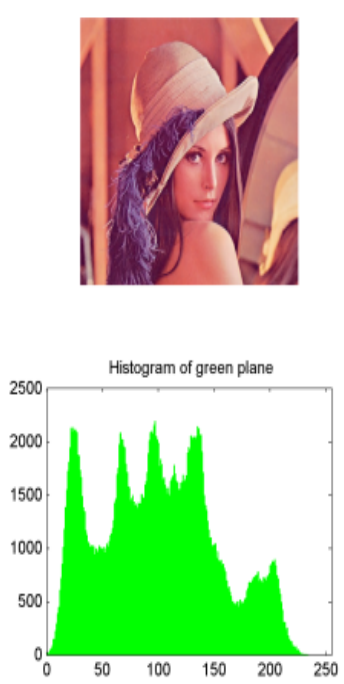
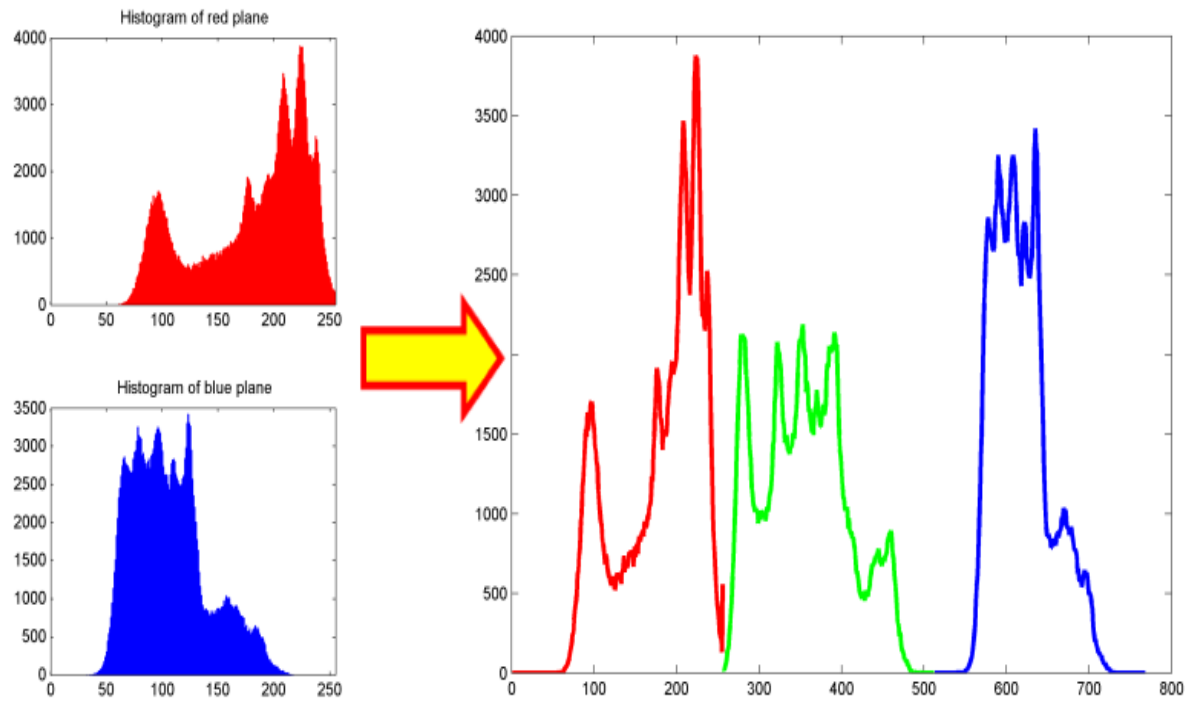

Figure 5. Color image histograms

\subsection{Color Imaage Feature Extraction Using CSLBP Method}

Calculation LBP operators depend on the neighbor's pixels values [9]. In the LBP method, an array of 256 elements (index) will be generated representing the image features. The LBP 256 operator can be calculated using the values of the neighbors of a pixel in the image as shown in Figure 6. 


\begin{tabular}{|c|c|c|c|c|c|c|}
\hline 100 & 120 & 200 & \multirow{7}{*}{$\rightarrow$} & 0 & 0 & 1 \\
\hline 160 & 150 & 70 & & 1 & $\mathrm{x}$ & 0 \\
\hline 30 & 180 & 50 & & 0 & 1 & 0 \\
\hline \multicolumn{3}{|c|}{ LBP calculati } & & \multicolumn{3}{|c|}{$\angle B P=C$} \\
\hline \multirow{3}{*}{\multicolumn{3}{|c|}{$\begin{array}{l}\text { Compare } \\
\text { neighbours with } \\
150\end{array}$}} & & \multicolumn{3}{|c|}{ Decimal $=82$} \\
\hline & & & & & & \\
\hline & & & & & & \\
\hline
\end{tabular}

Figure 6. LBP calculation using Neighbor pixels

However, LBP method does not reduce the histogram victor size (256 elements), and therefore it might not be suitable to extract color image features [8-11]. The CSLBP method creates a repetition value (from 0 to 15) for each pixel in the image; these repetitions can be used as a feature array to identify the image [17]. CSLBP method is used to generate features array for each pixel in the region [23], [24]. In CSLBP, center-symmetric pairs of pixels are compared to produce more compact binary patterns as shown in Figure 7. It decreases the number of features, thus increases the system efficiency.

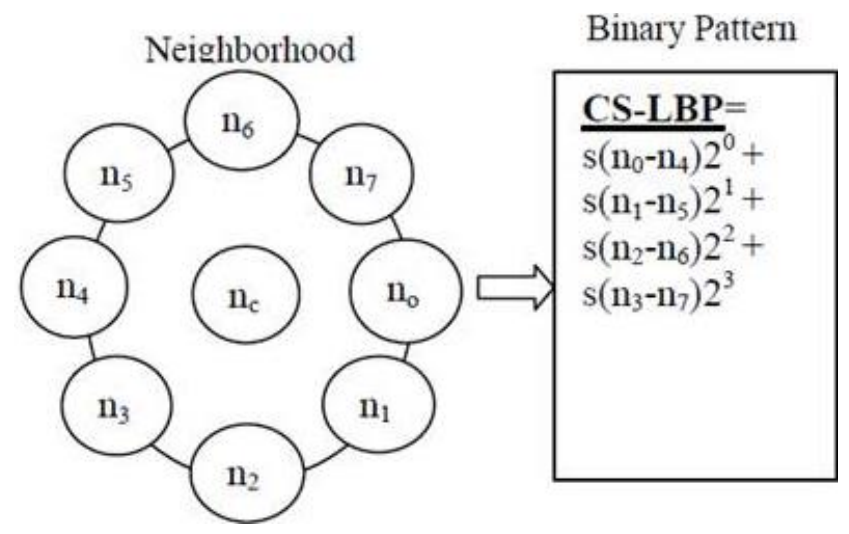

Figure 7. CSLBP calculation

An example is shown in Figure 7, in which 8 neighbors (n0 - n7), CS-LBP produced 16 different binary patterns. A Small threshold value was then used to obtain the robustness on flat image regions as shown in Figure 8(a).

Here, a matlab code was used and implemented to generate image features using CSLBP method. The results indicated that each feature array for each individual image is unique; accordingly, an array can be used as a key to identify its associated image. Figure 8(b), shows samples of the image features obtained as a result of implementing the matlab code (one column array for each color image). 
Color image
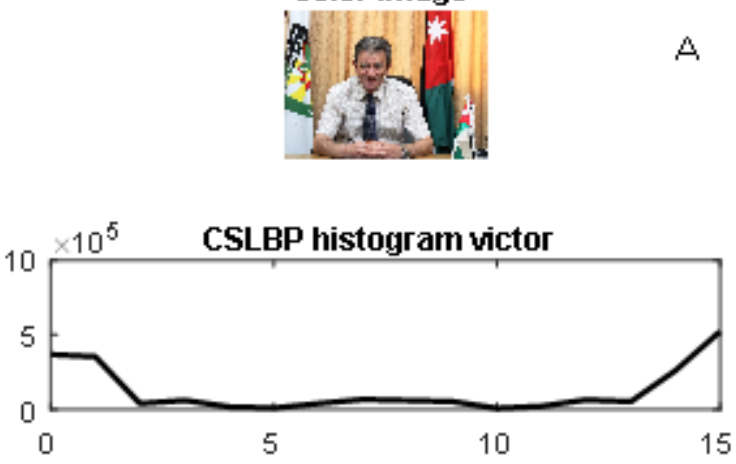

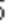

\begin{tabular}{|c|c|c|c|c|c|c|c|c|c|}
\hline 44920 & 28694 & 9089 & 211947 & 94738 & 579040 & 364147 & 164497 & 78080 & $\ldots 56$ \\
\hline 40941 & 33681 & 5747 & 102697 & 53709 & 394427 & 249463 & 97468 & 40005 & 16992 \\
\hline 4395 & 2670 & 1421 & 30977 & 15849 & 98198 & 64822 & 21299 & 14466 & $\$ 258$ \\
\hline 9594 & 3643 & 1802 & 56873 & 23831 & 156400 & 94780 & 52290 & 16406 & 6570 \\
\hline 2474 & 839 & 911 & 35009 & 14684 & 43663 & 37068 & 20676 & 10699 & 3764 \\
\hline 1982 & 819 & 671 & 17718 & 7132 & 34615 & 29842 & 6602 & 7350 & 2465 \\
\hline 4728 & 2210 & 1377 & 27596 & 14504 & 95740 & 59677 & 26028 & 12154 & 4380 \\
\hline 10099 & 3937 & 1967 & 66963 & 25675 & 185344 & 114437 & 52700 & 20997 & 7054 \\
\hline 7301 & 3800 & 1864 & 70998 & 23013 & 141004 & 8e872 & 47852 & 19376 & 6303 \\
\hline 6346 & 350 s & 1352 & 31735 & 15473 & 111151 & 71703 & 25894 & 12605 & 4301 \\
\hline 1441 & 652 & 639 & 17522 & 7167 & 30515 & 25521 & 7304 & 7689 & 2574 \\
\hline 3201 & 934 & 951 & 34502 & 14022 & 63340 & 47506 & 20374 & 11597 & 3990 \\
\hline 7897 & 3677 & 1814 & 59995 & 24361 & 134328 & 91243 & 60323 & 17031 & 6253 \\
\hline 6284 & 3638 & 1626 & 36351 & 18119 & 126714 & 85834 & 25260 & 15673 & 5510 \\
\hline 29455 & 19944 & 5932 & 98374 & 51057 & 349087 & 233008 & 100417 & 41143 & 14286 \\
\hline 87612 & 37428 & 10949 & 221933 & 133670 & 844435 & 694821 & 195524 & 103913 & 31585 \\
\hline
\end{tabular}

Figure 8. A: CSLBP histogram victor; B: Image feature samples obtained by CSLBP

\subsection{Image Locat Contrast Victor}

Image local contrast (LC) was proposed in 1992 by Hunt [25]. LC is an average difference between neighboring pixels, which can be used to generate local contrast array of 9 elements. Calculation of the LC involves the following steps:

a. Calculation of the scaled and corrected values of linear luminance Equation 1:

$$
l=\left(\frac{k}{255}\right)^{\gamma}
$$

Where: $k$ is the pixel value ( 0 to 255$), \gamma$ correction is 2 .

b. Calculation of the perceptual luminance $L$ using Equation 2:

$$
L=100 * \sqrt{l}=100 * \sqrt{\left(\frac{k}{255}\right)^{\gamma}}
$$

c. Calculating $l_{C i}$ for each of the following resolution levels (Resolutions=[1 2248162550100200$]$ using Equation 3.

$$
l_{c i}=\frac{\left|L_{i}-L_{i-1}\right|+\left|L_{i}-L_{i+1}\right|+\left|L_{i}-L_{i-w}\right|+\left|L_{i}-L_{i+w}\right|}{4}
$$

Figure. 10 explains the process of abstaining LC using 4 neighbors [22]. 


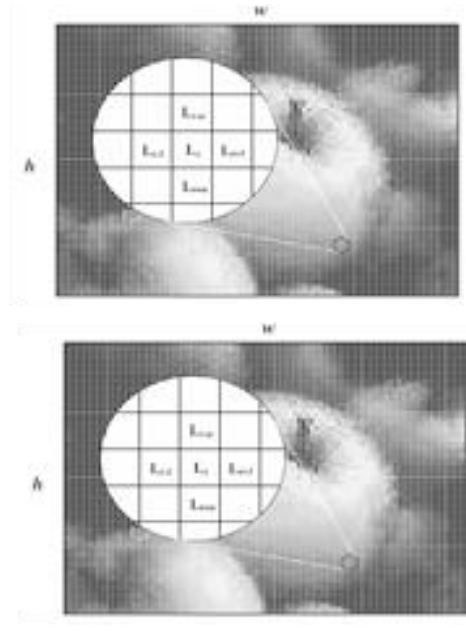

Figure 10. Using 4-neighbores to calculate LC

\section{IMPLEMENTATION}

To calculate the LC and to use LC array as an image features, the following steps were performed [9], [10]:

a. Get the original color image.

b. The color image matrix was reshaped from 3D to 2D.

c. Steps used to calculate the LC array were applied.

d. The obtained LC array was saved as a key for image identification.

\subsection{Experimental Results}

The following matlab function was written and implemented using various color images with different types and sizes:

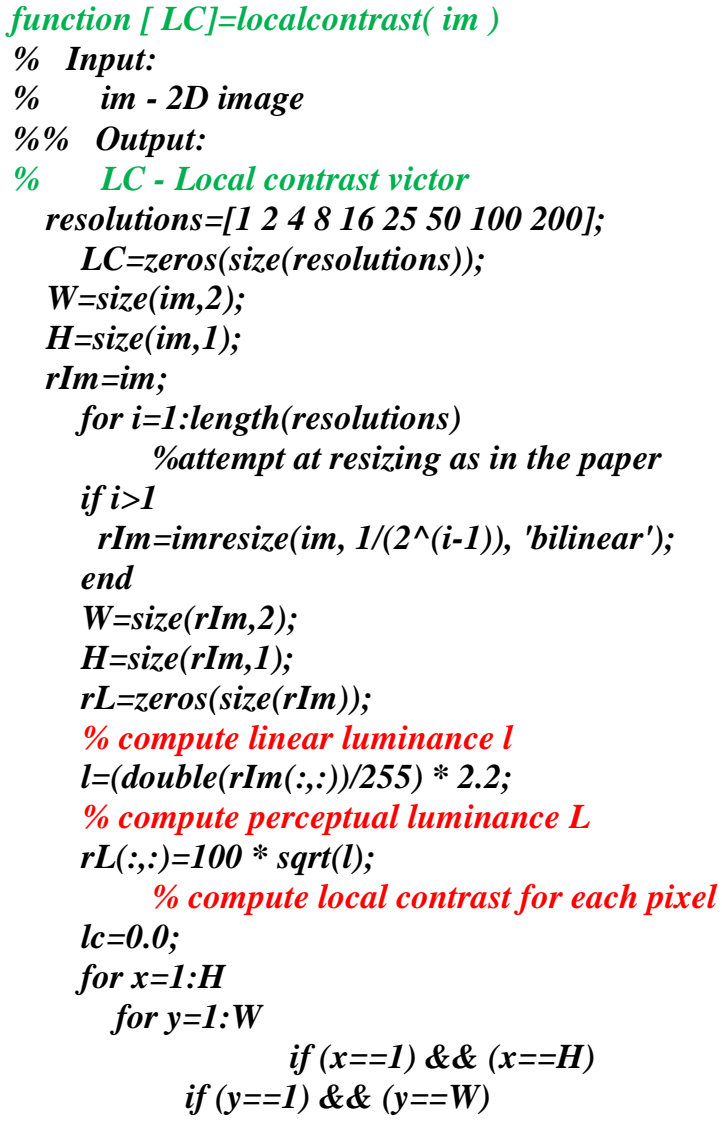




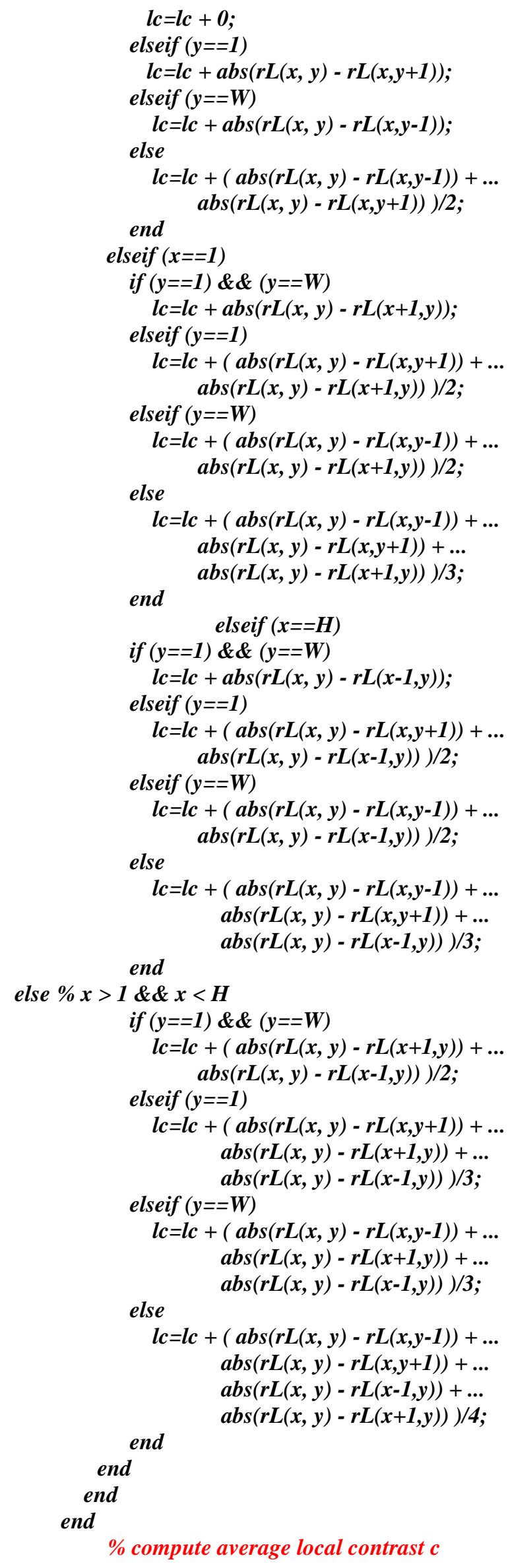




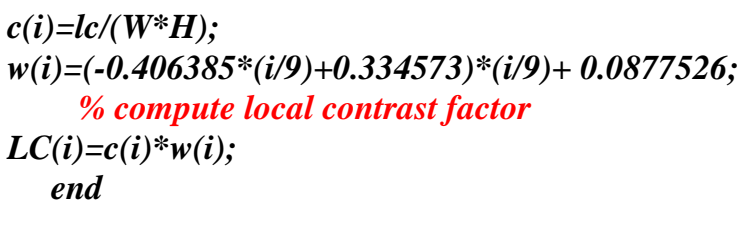

Different images were processed, and for each image in the dataset the LC array was obtained. The implementation results are shown in Table 1. From Table 1, it can be noticed that each LC array is unique, and thus it can be used as a key to identify the image.

Table 1. LC Array for Different Color Images

\begin{tabular}{cc}
\hline Image & Local contrast \\
\hline 1 & 0.7029 \\
2 & 0.7521 \\
3 & 1.0355 \\
4 & 0.9834 \\
5 & 1.4120 \\
6 & 0.8420 \\
7 & 0.6520 \\
8 & 1.2015 \\
9 & 1.2783 \\
10 & 1.7832 \\
\hline
\end{tabular}

Again and as abovementioned, LC array is unique and very sensitive to any changes in the original image, for example in the case the original image is changed, another and different LC array will be generated, which proves the uniqueness of LC array for each image, or for same image with slight changes and updates. Table. 2 shows different LC arrays for the same image with different versions (slight changes have been applied to the original image).

Table 2. Images Changes Lead to Changes in LC array

\begin{tabular}{cccccccccc}
\hline Image & \multicolumn{1}{c}{ Features } \\
\hline Origin & 0.1346 & 0.2283 & 0.3534 & 0.5632 & 0.8658 & 1.0041 & 0.8788 & 1.4651 & 0 \\
Changing pixel & 0.1349 & 0.2284 & 0.3533 & 0.5630 & 0.8654 & 1.0041 & 0.8788 & 1.4651 & 0 \\
Changing 2 pixels & 0.1351 & 0.2285 & 0.3533 & 0.5631 & 0.8654 & 1.0041 & 0.8788 & 1.4651 & 0 \\
\hline
\end{tabular}

The calculation time needed to generate the LC array was obtained by implementing the previous matlab function. Results are shown in Table 3.

Table 3. LC Calculation Time

\begin{tabular}{ccc}
\hline Image & Size (Pixels) & Calculation time (Seconds) \\
\hline 1 & 270948 & 0.631241 \\
2 & 151875 & 0.125924 \\
3 & 49152 & 0.101759 \\
4 & 1125600 & 0.841033 \\
5 & 540000 & 0.678468 \\
6 & 3396069 & 1.451037 \\
7 & 2359296 & 1.176620 \\
8 & 928800 & 0.784822 \\
9 & 432000 & 0.690299 \\
10 & 151353 & 0.128947 \\
\hline
\end{tabular}

\section{RESULTS COMPARISON}

A matlab code was written to create color images features using CSLBP method. Table 4 shows the extraction time for both methods. The comparison shows that the extraction time using CSLBP is lower compared to the time when LC extraction method was used. 
Table 4. Features Extraction Time

\begin{tabular}{ccc}
\hline Image & CSLBP extraction time (seconds) & LC calculation time (seconds) \\
\hline 1 & 0.0883 & 0.631241 \\
2 & 0.0481 & 0.125924 \\
3 & 0.0194 & 0.101759 \\
4 & 0.3390 & 0.841033 \\
5 & 0.1549 & 0.678468 \\
6 & 0.9680 & 1.451037 \\
7 & 0.6615 & 1.176620 \\
8 & 0.2636 & 0.784822 \\
9 & 0.1231 & 0.690299 \\
10 & 0.0477 & 0.128947 \\
Average & 0.2714 & 0.6610 \\
\hline
\end{tabular}

A dataset of 10 images features for each method was built, the ANN for each method was created and trained to achieve $100 \%$ recognition ratio and then tested to identify the image. Table. 5 summarizes comparison results. The results in Table. 5 clearly indicate the efficiency of the proposed local contrast LC method which exceeds the efficiency of CSLBP method.

Table 5. Results Comparisons

\begin{tabular}{ccc}
\hline Factor & LC method & CSLBP method \\
\hline Extraction time (average) & 0.6610 & 0.2714 \\
ANN training time (seconds) & 0.997369 & 9.311232 \\
Retrieving time (seconds) & 0.104247 & 0.120581 \\
ANN architecture & 3 layers with 9, 3, 1 neuron & 3 layers with 16, 4, 1 neuron \\
ANN activation functions & Tansig, tansig, linear & Tansig, tansig, linear \\
Number of features & 9 & 16 \\
Memory size to save ANN & $288 \mathrm{~KB}$ & $304 \mathrm{~KB}$ \\
Input data base size(10 images) & 720 byte & 1280 byte \\
\hline
\end{tabular}

\section{CONCLUSION}

In the current study, a local contrast method of color image features extraction was proposed. For each image, the obtained feature victor is unique, thus it can be used as a key to identify or retrieve the image. The proposed method was implemented and tested. The results showed that LC method can increase the system efficiency and performance compared with CSLBP method.

\section{REFERENCES}

[1] Uma S, Srujana B. Feature Extraction for Human Detection using HOG and CS-LBP methods. International Journal of Computer Applications (0975 - 8887) National Conference Electronics, Signals, Communication and Optimization, NESCO-2015: 11-14.

[2] Yang S, Liao X, Borasy U. A Pedestrian Detection Method Based on the HOG-LBP Feature and Gentle AdaBoost. International Journal of Advancements in Computing Technology. 2012; 4(19): 553-560.

[3] Choras, R. Image feature extraction techniques and their applications for CBIR and biometrics systems. International Journal of Biology and Biomedical Engineering. 2007; 1(1): 6-16.

[4] Matrouk K, Alasha'ary H, Al-Hasanat A, Al-Qadi Z, Al-Shalabi H. Investigation and Analysis of ANN Parameters. European Journal of Scientific Research. 2014; 121(2): 217-225.

[5] Kohonen T. An Introduction to Neural Computing. Neural Networks. 1988; 1(1): 3-16.

[6] Hecht-Nielsen R. Counter-Propagation Networks. Proceedings of the IEEE First International Conference on Neural Networks. Ssan Diego, CA, USA. 1987; 19-32.

[7] Lu T, Chang C. Color image retrieval technique based on color features and image bitmap. Information processing \& management. 2007; 43(2): 461-72.

[8] Hong X, Zhao G, Pietikainen M, Chen X. Combining LBP difference and feature correlation for texture description. IEEE Transactions on Image Processing, 2014; 23(6): 2557-2668.

[9] Qaryouti G, Khawatreh S, Alqadi Z, Abu Zalata M. Optimal Color Image Recognition System (OCIRS). International Journal of Advanced Computer Science and Technology, 2017; 7(1): 91-99.

[10] Gupta R, Patil H, Mittal A. Robust order-based methods for feature description. IEEE conference on computer vision and pattern recognition (CVPR). San Francisco, CA, USA. 2010.

[11] Rami H, Hamri M, Masmoudi L. Objects Tracking in Images Sequence Using Center-Symmetric Local Binary Pattern (CS-LBP). International Journal of Computer Applications Technology and Research. 2013; 2(5): 504 508.

[12] Kong H, Wang L, Teoh E, Li X, Wang J, Venkateswarlu R. Generalized 2D principal component analysis for face image representation and recognition. Neural Networks. 2005; 18(5-6): 585-594. 
[13] Abu Zneit R, AlQadi Z, Abu Zalata M. A Methodology to Create a Fingerprint for RGB Color Image. Internationa Journal of Computer Science and Mobile Computing. 2017; 6(1): 205 - 212.

[14] Murala S, Maheshwari R, Balasubramanian R. Local tetra patterns: a new feature descriptor for content-based image retrieval. IEEE Transactions on Image Processing. 2012; 21(5): 2874-2886.

[15] Leon K, Mery D, Pedreschi F, Leon J. Color measurement in L* $\mathrm{a}^{*} \mathrm{~b}^{*}$ units from RGB digital images. Food Research International. 2006; 39(10): 1084-1091.

[16] Yam K, Papadakis S. A simple digital imaging method for measuring and analyzing color of food surfaces. Journal of Food Engineering. 2004; 61(1): 137-142.

[17] Heikkilä M, Pietikäinen M, Schmid C. Description of Interest Regions with Center-Symmetric Local Binary Patterns. ICVGIP-LNCS, 2006; 4338: 58-69.

[18] Petruk V. Application of Local Binary Patterns to Face Recognition Problem Solving. Joint Advanced Student School, Bauman Moscow State Technical University- Moscow, Russia, 2011.

[19] Gehler P, S. Nowozin. On feature combination for multiclass object classification. IEEE International conference of Computer Vision. Kyoto, Japan. 2009: 221-228.

[20] Henry C, Peters J. Perceptual image analysis. International Journal of Bio-Inspired Computation. 2010; 2(3/4): 271281.

[21] Li J, Zhao Y, Quan D. The combination of CSLBP and LBP feature for pedestrian detection. Computer Science and Network Technology (ICCSNT), 3rd International Conference, Dalian, India. 2013: 543-546.

[22] Matković K, Neumann L, Neumann A, Psik T, Purgathofer W. Global Contrast Factor - a New Approach to Image Contrast. Computational Aesthetics in Graphics, Visualization and Imaging, 2005; 18-20.

[23] Baber J, Satoh S, Keatmanee C, Afzulpurkar N. Improving the performance of SIFT and CSLBP for image copy detection. Telecommunications and Signal Processing (TSP), 36th International Conference. Rome, Italy. 2013; 803-807.

[24] Ojala T, Pietikainen M, Maenpaa T. Multiresolution gray-scale and rotation invariant texture classification with local binary patterns. IEEE Transactions on pattern analysis and machine intelligence. 2002; 24(7): 971-987.

[25] Hunt R. Measuring Color", 2 $2^{\text {nd }}$ edition. Ellis Horwood, 1996. 